\title{
Prioritization-based Layered Multicast for Fixed/Mobile Networks with Fast Convergence and Inter-Session Fairness
}

\author{
Kenichi Kashibuchi, Tarik Taleb, Abbas Jamalipour, Yoshiaki Nemoto, and Nei Kato
}

\begin{abstract}
Multimedia streaming services are becoming popular in both wired and wireless networks. Layered multicast is a widely accepted approach for streaming multimedia data to a large number of users. Existing layered multicast approaches do not interact well with network dynamics. Indeed, upon a change in network conditions, they require a long time till they can appropriately adjust their data transmission rate. Additionally, they do not achieve fairness when users from different sessions share the bandwidth of a bottleneck link.

In this paper, we propose a scheme that allows newly-arriving users to promptly converge their data transmission rates to the most optimal rate that best suit the current conditions of the network without degrading the system fairness. The proposed scheme is based on the fact that layered multicast uses prioritybased packet dropping policies. In the proposed scheme, two newly-defined packet messages are considered: "low priority join" and "normal join" messages. To join a session, a user first subscribes to all corresponding layers by issuing "low priority join" messages. It then computes packet drops experienced on each layer. If packets of a given layer experience a drop rate higher than a predetermined threshold, the user leaves that layer and all higher layers. The user then "officially" joins the remaining layers by transmitting "normal join" messages. This operation helps users to subscrive to only layers whose aggregate bandwidth fits the current network conditions.

The performance of the proposed scheme is evaluated through computer simulations and is compared against the Receiverdriven Layered Multicast (RLM) scheme. The results show that the proposed scheme achieves appropriate bandwidth utilization from the start of the session. The results demonstrate also that the proposed scheme is effective in managing handover in mobile networks and achieves better Quality of Service (QoS) in heterogeneous mobile environments.
\end{abstract}

Index Terms - layered multicast, priority dropping, QoS, handoff management

\section{INTRODUCTION}

Along with the recent advances and on-going improvements in broadband Internet access technologies, a plethora of wide-band multimedia services has appeared. Simultaneous streaming of such services to a potentially large number of users is a challenging task for current unicast-based Internet

Manuscript received February 28, 2006 and revised May 04, 2006.

K. Kashibuchi, T. Taleb, Y. Nemoto, and N. Kato are with the Graduate School of Information Sciences, Tohoku University, Sendai, Japan (e-mail: buchiken@it.ecei.tohoku.ac.jp; talebtarik@ieee.org; nemoto@ nemoto.ecei.tohoku.ac.jp; kato@it.ecei.tohoku.ac.jp)

A. Jamalipour is with the School of Electrical and Information Engineering, University of Sydney, Australia (e-mail: a.jamalipour@ieee.org). technologies. Multicast is seen as an attractive solution for large-scale streaming of these multimedia services. Whilst multicast is optional in existing Internet Protocol version 4 (IPv4) and is not widely spread, in next generation Internet Protocol, Internet Protocol version 6 (IPv6), all hosts and routers are required to support multicast. Therefore, it seems that realtime streaming will use multicast along with the spread of IPv6.

On the other hand, current wireless accesses, such as Wireless LAN (WLAN), Worldwide Interoperability for Microwave Access (WiMAX), and $3^{\text {rd }}-4^{\text {th }}$ generation cellular systems $(3-4 \mathrm{G})$, enable broadband communications in mobile networks. Along with further development of such access technologies, mobile users (laptop computers with wireless interfaces for WLAN or mobile phones) may desire to receive multimedia services while they are on move.

However, the progress of such various Internet access technologies means that users may have to access different networks with different available bandwidths. For instance, a user may access the Internet at nearly $100 \mathrm{Mbps}$ (e.g. via a Fiber To The Home (FTTH) line) and another user may access the Internet at a few Mbps (e.g. via a cellular network). In general IP multicast, since the server streams data at only a single rate, multimedia data are transmitted with same contents and same quality (bit rate) for all users. Accordingly, in order to deliver data to as many users as possible, streaming rates should take into account the available bandwidths of users. Indeed, users with enough available bandwidth should receive data at high rates and users with lower available bandwidth should receive data at low quality (low bit rate).

In order to deal with such network diversity, the cumulative layered multicast approach has been proposed [1] [2]. In layered multicast, streaming servers encode the multimedia data in a base layer and several other enhancement layers. They transmit each layer on a different multicast channel. In case of receiver-driven congestion control, receivers assess whether to add or drop layers (i.e. to join or leave a multicast group) according to the network conditions. Receivers first join the base layer. They then join the upper enhancement layers in sequence if the network conditions allow. In layered encoding, the more layers users receive, the higher quality they can get. However, since the enhancement layers depend on lower layers, the user cannot decode data without lower layers. If packets of some lower layers are dropped in the 
network, packets from higher layer become useless. As a result, the effective packet loss rate becomes high. Therefore, since lower layers provide more important data and should be given higher priority, routers dealing with layered multicast approaches should be equipped with a priority-based packet dropping policy [3][4]. Among receiver-driven layered multicast approaches, Receiver-driven Layered Multicast (RLM) is a notable example [2]. A major drawback of RLM consists in the following. In the beginning of a session, users join the base layer and then join the higher enhancement layers one-byone. Therefore, RLM requires a long time until an appropriate multicast group is found. Additionally, RLM does not have inter-session fairness.

Moreover, when providing multimedia streaming services to mobile users, users' mobility should be considered. Indeed, when a mobile node performs handoff between two base stations (access points), if the two cells have different available bandwidths, the user cannot receive video data at a rate suitable for the new cell immediately after the handoff. This bandwidth disparity can be due to the difference in traffic load in both wireless cells or use of different wireless access techniques with different link speeds, such as WLAN, 3G, $4 \mathrm{G}$, or WiMAX. If the user moves from a higher bandwidth network (e.g. WLAN) to a lower bandwidth network (e.g. $3 \mathrm{G}$ ) without changing the total number of layers (i.e. bit rate), the new network may be congested and a number of packets may be dropped. This will eventually affect the streaming quality. On the other hand, if the mobile node moves from a lower bandwidth network to a higher bandwidth network, no adjustment to the streaming rate will degrade the service quality in an environment where higher quality can be obtained. This results in a waste of the network resources.

To solve these issues, this paper proposes a scheme that quickly converges to the most appropriate rate while guaranteeing fairness among all sessions. The mechanism of the proposed scheme is extended further to mobile networks by taking into account the mobility of users.

The remainder of this paper is structured as follows. Section II surveys recent research work on layered multicast techniques for multimedia streaming. Section III introduces the proposed scheme. Following this, Section IV describes the simulation setup and discusses the simulation results. Finally, the paper concludes in Section V.

\section{RELATED WORK}

To tackle issues related to network diversity, two types of multicast streaming can be envisioned: replicated streaming and layered streaming. In the former [5], which is also called as simulcast approach, a video server transmits multiple streams carrying the same data with different qualities (different bit rates). Each stream is multicast on a different multicast channel. Each user joins the multicast group that best satisfies its needs. In the latter, the server divides its data into layers: a base layer and several other enhancement layers. It transmits each layer to a different multicast group. The base layer can be independently decoded and provides the basic-level quality. The enhancement layers can be decoded together with the base layer. Users join the base layer group and join as many enhancement layer groups as the network condition permits. In general, the layered multicast approach is more advantageous than the simulcast approach in bandwidth utilization, especially in highly heterogeneous networks. A detailed description of the two approaches can be founded at [6].

Transcoding is another technique for multicast streaming [7]. In this approach, video streams are filtered or transcoded to the optimal rate at routers, application gateways, or wireless base stations in the network. A trivial drawback of this approach is that it causes significant load to these network elements.

Among layered multicast approaches, RLM and packet Pair receiver-driven cumulative Layered Multicast (PLM) [8] are notable examples. In RLM, a server transmits data packets of each layer using different multicast group addresses and users join as many layers as their available bandwidths allow. If a user has sufficient bandwidth, he/she joins the next enhancement layer. In contrast, if he/she detects network congestions, he/she quits receiving the highest enhancement layer. This procedure is called "join-experiments". At the beginning of a session, RLM assesses its available bandwidth in a slow start-like fashion. That is, users converge to the optimal rate by subscribing to next enhancement layers incrementally until congestion occurs (i.e. packet losses). Therefore, it needs a long convergence time. Furthermore, joining higher layers may yield packet losses and may degrade the streaming quality. In addition, since old users do not change their groups, newly-arriving users may not find sufficient bandwidth. This ultimately yields unfairness among users.

To solve these issues, PLM has been proposed. PLM estimates the available bandwidth based on a packet pair mechanism without join-experiments. All packets on all layers are transmitted in pairs. At the beginning of a session, users simply join the base layer. Users then estimate their available bandwidth $C$ seconds ( $C=1$ in [8]) after receiving the first packet pair and check whether they should add or drop layers. Therefore, PLM can converge to the optimal rate faster than RLM, without inducing losses. However, PLM requires that all routers in the network should implement a fair scheduler. Moreover, as the bandwidth estimation is based on an accurate estimation of propagation delays, PLM may fail in the bandwidth estimation in case the network load becomes heavy.

On the other hand, in mobile networks, users freely perform handoff upon changing their point of attachment to the network. To guarantee smooth handoff, several approaches have been proposed. In [9] multiple paths are established between the server and a mobile node during handoff. Admittedly this scheme provides smooth handoff for streaming media. Nevertheless it uses multiple paths during the time a mobile node exists in the cell overlapping area. As in the case of a handoff between $3 \mathrm{G}$ and WLAN, if the distance of the cell overlapping area is long, it is unacceptable to use multiple paths for a long time as it causes redundant transmissions of important data. Although it does not consider the layered multicast approach, the research work outlined in [10] realizes a seamless handoff for streaming video by using two simultaneous connections 
through two separate WLANs. The handoff occurrence time is computed based on the delay difference. This approach is however effective for only horizontal handoffs and not vertical ones.

\section{Proposed SCHEME}

This section describes in detail the proposed scheme. It first presents the preconditions that are required for the implementation of our scheme.

\section{A. Preconditions}

The proposed scheme is based on the cumulative layered streaming approach, similarly to RLM and PLM. As in any layered streaming mechanism, all routers are assumed to be multicast-capable and to support some priority-based dropping disciplines.

Input multimedia data are encoded into a set of $n$ cumulative layers $\left(L_{1}, \ldots, L_{n}\right)$. All subsets $\left\{L_{1}, \ldots, L_{i}\right\}_{i<n}$ provide the same content. The provided quality increases as index $i$ increases. Since the lowest layer contains the most important data, packets from the lowest layer are assigned higher priority. Indeed, the base layer $L_{1}$ has the highest priority (the lowest drop probability) among all layers. Higher layers have lower priority as index $i$ increases. Let $B_{i}$ and $P_{i}$ denote the bit rate and the priority of layer $L_{i}$, respectively. $P_{i}$ depends on the aggregate bandwidth of the $(i-1)$ cumulative layers $B_{i-1}^{c}$ :

$$
B_{i-1}^{c}= \begin{cases}\sum_{k=1}^{i-1} B_{k} & \text { for } i \in\{2, \ldots, n\} \\ 0 & \text { for } i=1\end{cases}
$$

We assign these priorities at the streaming servers using the class field in the IPv6 packet header. Similarly in spirit to the Assured Forwarding (AF) class of the DiffServ CodePoint (DSCP) [11], we consider the setup of twelve priority levels. An example of this priority setting is given in Table I. In this example the values of $P_{i}$ are calculated as follows:

$$
P_{i}= \begin{cases}12-\max \left(0,\left\lfloor\log _{2} \frac{B_{i-1}^{c}}{10^{4}}\right\rfloor\right) & \text { for } i \in\{2, \ldots, n\} \\ 12 & \text { for } i=1\end{cases}
$$

In the proposed scheme, two signaling packets are defined: "low priority join" and "normal join". The former is transmitted by users to local multicast routers in order to join a

TABLE I

AN EXAMPLE OF PRIORITY SETTING.

\begin{tabular}{c|c|c}
\hline \hline Priority $P_{i}$ & DSCP & Cumulative Rate $B_{i-1}^{c}$ \\
\hline 12 & AF41 & $<20 \mathrm{kbps}$ \\
11 & AF42 & $<40 \mathrm{kbps}$ \\
10 & AF43 & $<80 \mathrm{kbps}$ \\
9 & AF31 & $<160 \mathrm{kbps}$ \\
8 & AF32 & $<320 \mathrm{kbps}$ \\
7 & AF33 & $<640 \mathrm{kbps}$ \\
6 & AF21 & $<1.25 \mathrm{Mbps}$ \\
5 & AF22 & $<2.5 \mathrm{Mbps}$ \\
4 & AF23 & $<5 \mathrm{Mbps}$ \\
3 & AF11 & $<10 \mathrm{Mbps}$ \\
2 & AF12 & $<20 \mathrm{Mbps}$ \\
1 & AF13 & $\geq 20 \mathrm{Mbps}$ \\
\hline \hline
\end{tabular}

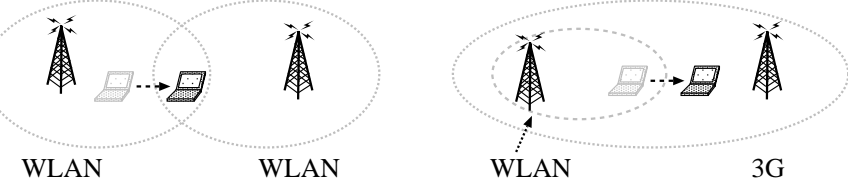

Fig. 1. Two different scenarios of handoff.

session or conduct join-experiments. Upon receiving a low priority join message, multicast routers subsequently decrease the priority of the forwarded streaming packets by one and forward them. In other words, the priority of layer $L_{i}\left(P_{i}\right)$ is decremented to $\left(P_{i}-1\right)$, not $P_{i-1}$. These streams with lower priority do not affect the network because their packets are discarded first when a downstream router is congested. A normal join message is transmitted by users to local multicast routers in order to receive packets with normal priority $P_{i}$. After receiving a normal join message, multicast routers forward packets without changing their priority.

All multicast routers maintain an internal table with information on source address, multicast group address, outgoing interface identifier, and priority level. In the table, the priority field can be set to either " $N$ " or " $L$ ". $L$ indicates that the priority of packets is decreased by one by this multicast router, while $N$ indicates that packets are forwarded without changing their priority.

Additionally, when we apply our scheme to mobile users, it is assumed that wireless cells overlap with each other as shown in Fig. 1. To access two networks in parallel, a mobile node needs to be simply equipped with two wireless interfaces. This assumption is based on the work presented in [12], in which a single physical WLAN interface is used to simultaneously access multiple WLANs. Moreover, along with a further integration of wireless technologies, it will become normal for a mobile node to have an interface that can simultaneously access different types of wireless networks.

\section{B. Description of the proposed scheme}

As previously mentioned, a server $S$ encodes multimedia data into a set of $n$ cumulative layers $\left(L_{1}, \ldots, L_{n}\right)$. Layer $L_{i}$ is multicast to group $G_{i}$ with priority $P_{i}$. Lower layers are transmitted with higher priorities (i.e. $P_{i}>P_{i+1}$ ).

At the beginning of a session, users transmit "low priority join" messages $\left\{\left(S, G_{i}\right), i=1, \ldots, n\right\}$ to local multicast routers. Upon receiving a low priority join message $\left(S, G_{i}\right)$, multicast routers first check whether the stream corresponding to $L_{i}$ is flowing or not (i.e. whether the entry $\left(S, G_{i}, *^{1}, N\right)$ exists in the internal table). If a multicast router does not have the entry $\left(S, G_{i}, *, N\right)$, it adds the entry $\left(S, G_{i}, I F, N\right)$ to its table and forwards the low priority join message $\left(S, G_{i}\right)$ to its upper multicast router. Here, IF indicates the interface identifier that received the low priority join message. On the contrary, if the multicast router has the entry $\left(S, G_{i}, *, N\right)$, it adds the entry $\left(S, G_{i}, I F, L\right)$ to the table and subsequently decreases the priority of the forwarded packets from $P_{i}$ to $\left(P_{i}-1\right)$. It then transmits packets to the requesting users

\footnotetext{
${ }^{1}$ Asterisk $(*)$ denotes unspecified interface.
} 
or lower multicast routers. In this manner, multicast routers forward the low priority join message $\left(S, G_{i}\right)$ to upper routers until the expected stream is found.

After receiving the first packet of group $G_{i}$, which is transmitted with the lower priority $\left(P_{i}-1\right)$, users calculate the packet loss rate $\rho_{i}$ experienced by packets of layer $L_{i}$ for an interval of time $T_{D}$ (detection timer). This packet loss rate can be simply calculated from the sequence number available in the packet header of Real-time Transport Protocol (RTP) [13]. If $\rho_{k}$, the packet loss rate of $L_{k}$, is smaller than a predefined threshold $\theta$, users transmit a normal join message $\left(S, G_{k}\right)$ to the local multicast router. The setting of $\theta$ indicates the system tolerance level in terms of packet drops and depends on Forward Error Correction (FEC) redundancy. If $\rho_{k}$ is above $\theta$, the users stop receiving packets of layer $L_{k}$ and higher layers by leaving multicast groups $\left\{G_{i}, i=k, \ldots, n\right\}$.

Upon receiving a normal join message $\left(S, G_{k}\right)$, a multicast router verifies whether it has the entry $\left(S, G_{k}, I F, L\right)$ or not. In case it does not have the entry $\left(S, G_{k}, I F, L\right)$, it forwards the normal join message $\left(S, G_{k}\right)$ to the upper multicast router. On the contrary, when it has the entry $\left(S, G_{k}, I F, L\right)$, it modifies the entry from $\left(S, G_{k}, I F, L\right)$ to $\left(S, G_{k}, I F, N\right)$ to indicate that it is forwarding packets with normal priority $P_{k}$.

After a given period of time $T_{J}$ (join timer), users send low priority join message $\left(S, G_{k}\right)$ to perform the join-experiment operation with lower priority. After receiving the first packet of layer $L_{k}$, users calculate the packet loss rate $\rho_{k}$ on the layer $L_{k}$ for an interval of time $T_{D}$, as in the beginning of the session. When $\rho_{k}$ is smaller than the threshold $\theta$, users transmit a normal join message $\left(S, G_{k}\right)$ to the local multicast router and join $L_{k}$ with normal priority. In case $\rho_{k}$ exceeds $\theta$, users interpret this join-experiment as failure and leave layer $L_{k}$. They then multiplicatively increase the join timer $T_{J}$ as follows:

$$
T_{J} \leftarrow \alpha \cdot T_{J}
$$

where $\alpha$ is a constant. In the proposed scheme, $T_{D}$ and $T_{J}$ are set to random values uniformly chosen from $\left[0.5 \cdot T_{D}, 1.5 \cdot T_{D}\right]$ and $\left[0.5 \cdot T_{J}, 1.5 \cdot T_{J}\right]$, respectively. This setting aims to avoid simultaneous requests from different users.

\section{Explanation of the proposed scheme via an example}

In this section, we describe the procedures of the proposed scheme via an example using Figs. 2 and 3. The latter corresponds to the time interval $\left[t_{0}, t_{1}\right]$ in Fig. 2 . In this example, server $S$ encodes multimedia data into a set of four cumulative layers $\left(L_{1}, L_{2}, L_{3}\right.$, and $\left.L_{4}\right)$. Each layer $L_{i}$ is transmitted to multicast group $G_{i}$ with priority $P_{i}(i=1,2,3,4)$.

At time $t=0$, receiver $R_{1}$ joins a session from server $S$ and receives data from three layers (i.e. $L_{1}, L_{2}$, and $L_{3}$ ) (Fig. 3(a)). After a while, a new user $R_{2}$ joins the same session by issuing low priority join messages $\left\{\left(S, G_{i}\right), i=\right.$ $1,2,3,4\}$ to the local multicast router $\mathrm{MR}_{3}$. Since $\mathrm{MR}_{3}$ does not have any entry $\left(S, G_{i}, *, N\right)$, it adds the entries $\left\{\left(S, G_{i}, I F_{5}, N\right), i=1,2,3,4\right\}$ and forwards the low priority join messages $\left\{\left(S, G_{i}\right), i=1,2,3,4\right\}$ to its upper multicast router $\mathrm{MR}_{2} . \mathrm{MR}_{2}$ then adds the entries $\left\{\left(S, G_{m}, I F_{4}, L\right), m=\right.$ $1,2,3\}$ and $\left(S, G_{4}, I F_{4}, N\right)$, and forwards the low priority

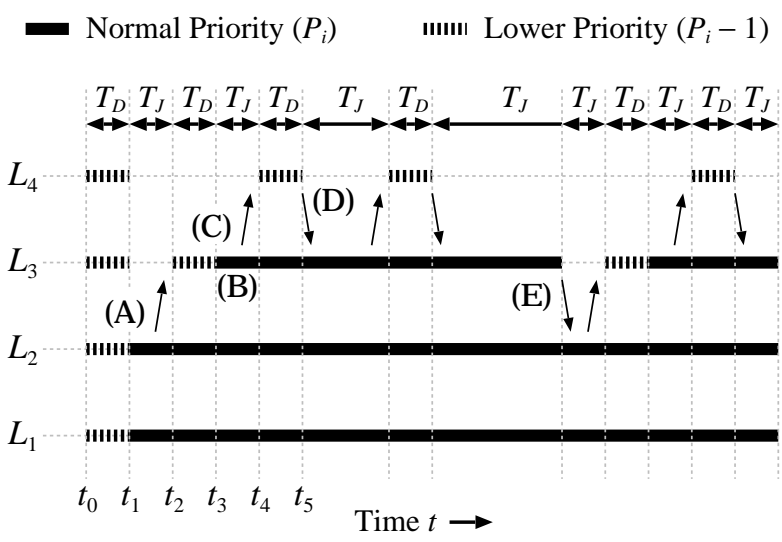

Fig. 2. An example of $R_{2}$ 's layer subscription.

join message $\left(S, G_{4}\right)$ to its upper multicast router $\mathrm{MR}_{1}$. In response, $\mathrm{MR}_{1}$ adds the entry $\left(S, G_{4}, I F_{1}, L\right)$ to its internal table (Fig. 3(b)). It then forwards packets of layer $L_{4}$ to receiver $R_{2}$ via $\mathrm{MR}_{2}$ and $\mathrm{MR}_{3}$ with lower priority. In its turn, $\mathrm{MR}_{2}$ transmits data of layers $L_{1}, L_{2}$, and $L_{3}$ to $R_{2}$ via $\mathrm{MR}_{3}$ also with lower priority (Fig. 3(c)).

We assume that $R_{2}$ receives the first packet of group $G_{i}$ at time $t=t_{0}$. At $t=t_{1}=\left(t_{0}+T_{D}\right), R_{2}$ calculates the packet loss rate $\rho_{i}$ on layer $L_{i}$. In this example, $\rho_{3}$ is assumed to be above the threshold $\theta$. Therefore, the user transmits a normal join messages $\left\{\left(S, G_{k}\right), k=1,2\right\}$ to $\mathrm{MR}_{3}$ and leaves $L_{3}$ and $L_{4}$. Since $\mathrm{MR}_{3}$ does not have the entries $\left(S, G_{k}, I F_{5}, L\right)$, it forwards the normal join messages $\left(S, G_{k}\right)$ to $\mathrm{MR}_{2}$ (Fig. 3(d)). In response, $\mathrm{MR}_{2}$ resets the entries $\left(S, G_{k}, I F_{4}, L\right)$ to $\left(S, G_{k}, I F_{4}, N\right)$. Afterwards, $\mathrm{MR}_{2}$ subsequently forwards the packets to $\mathrm{MR}_{3}$ without changing the priority (i.e. with $P_{k}$ ) (Fig. 3(e)).

At $t=t_{2}=\left(t_{1}+T_{J}\right), R_{2}$ transmits a low priority join message $\left(S, G_{3}\right)$ to perform a join-experiment with lower priority and receives data of $L_{3}$ with lower priority $\left(P_{3}-1\right)$ (Fig. 2(A)). At $t=t_{3}$, if the calculated packet loss rate $\rho_{3}$ is not larger than $\theta, R_{2}$ transmits a normal join message $\left(S, G_{3}\right)$ and joins $L_{3}$ with normal priority (Fig. 2(B)).

In a similar way, at $t=t_{4}, R_{2}$ transmits a low priority join message $\left(S, G_{4}\right)$ and receives $L_{4}$ with lower priority $\left(P_{4}-1\right)$ (Fig. 2(C)). At $t=t_{5}$, if $\rho_{4}$ exceeds $\theta, R_{2}$ stops receiving layer $L_{4}$ and $T_{J}$ is multiplicatively increased (Fig. 2(D)). After time $T_{J}, R_{2}$ performs again a join-experiment with lower priority. If $R_{2}$ infers a possible congestion occurrence from the loss rate of $L_{3}, R_{2}$ leaves $L_{3}$ (Fig. 2(E)).

\section{Handoff management in the proposed scheme}

In the remainder of this section, we explain how the proposed scheme can be applied in mobile networks. This application is similar in spirit to the idea presented in [14] [15]. Fig. 4 illustrates the idea. A mobile node (MN) instantly measures radio strength or link quality. Prior to handoff, $\mathrm{MN}$ receives data via base station $\mathrm{BS}_{1}$ using wireless interface $\mathrm{IF}_{1}$. When $\mathrm{MN}$ moves into the new cell of base station $\mathrm{BS}_{2}$, a new network address is given to the MN's wireless interface $\mathrm{IF}_{2}$. When radio strength or link quality through $\mathrm{IF}_{1}$ goes down 


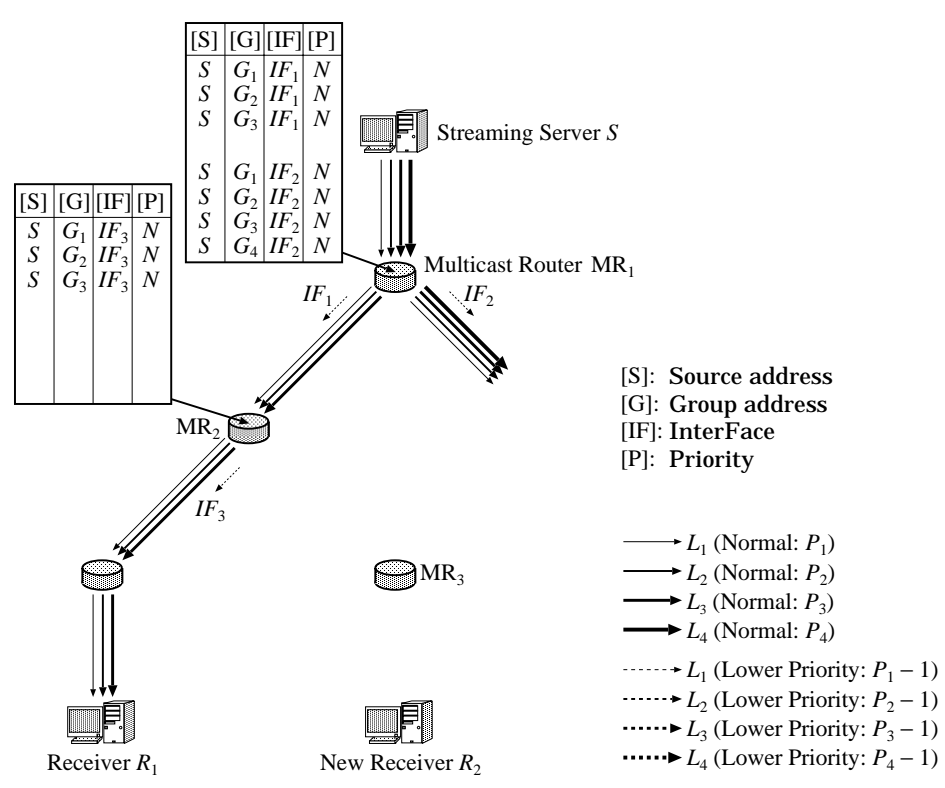

(a) Initial state when $R_{1}$ is receiving the session.

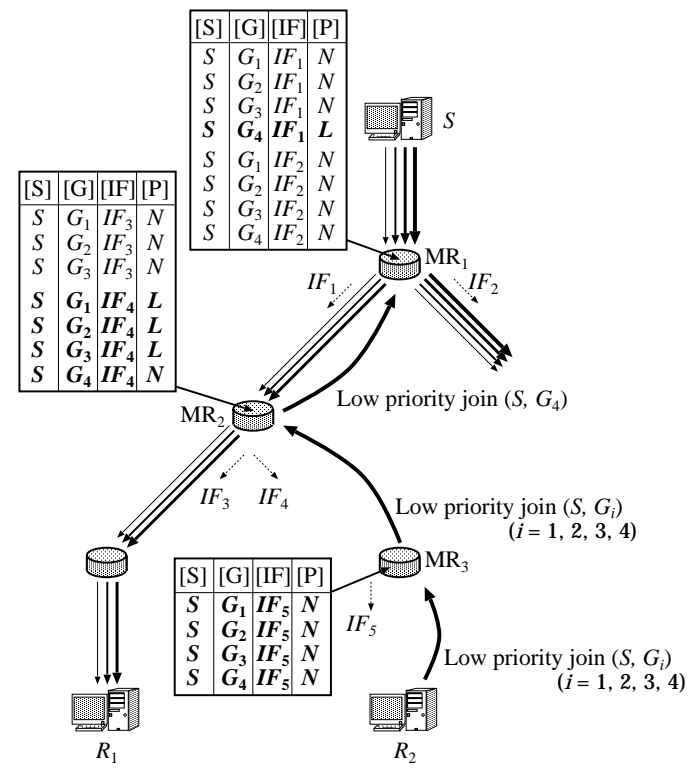

(b) $R_{2}$ joins the session with lower priority.

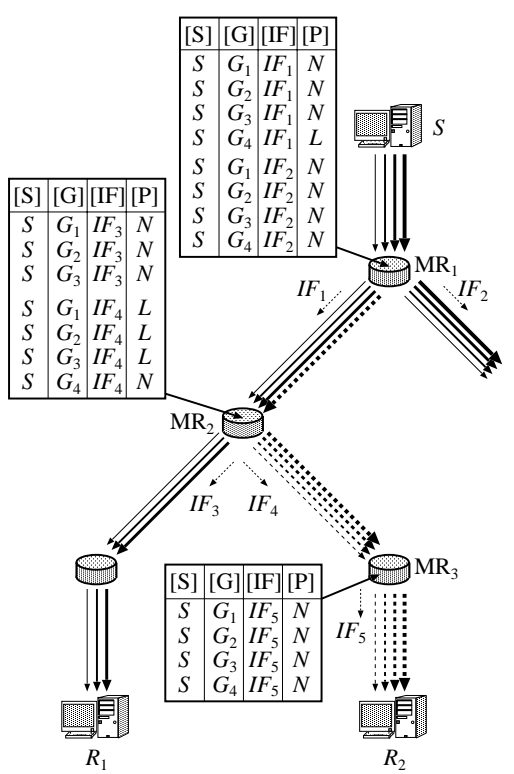

(c) $R_{2}$ receives stream with lower priority.

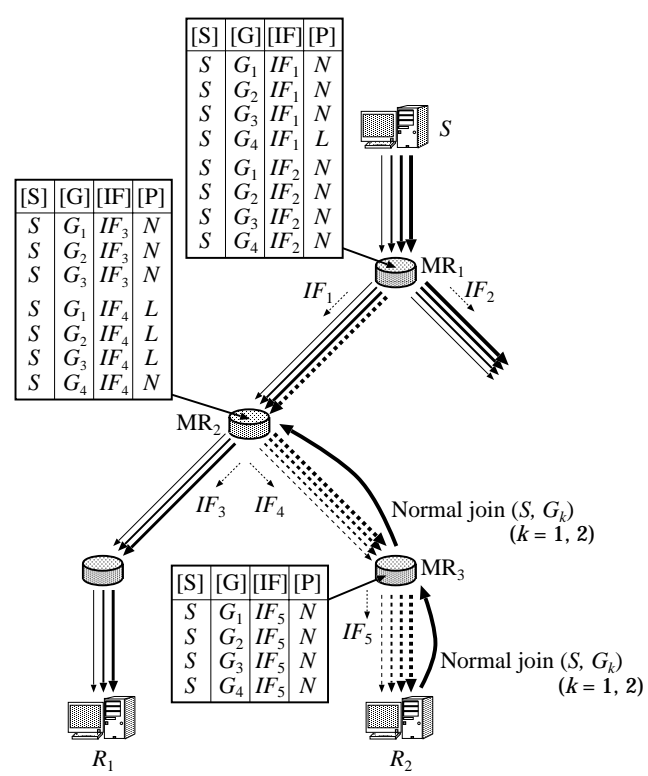

(d) $R_{2}$ transmits normal join.

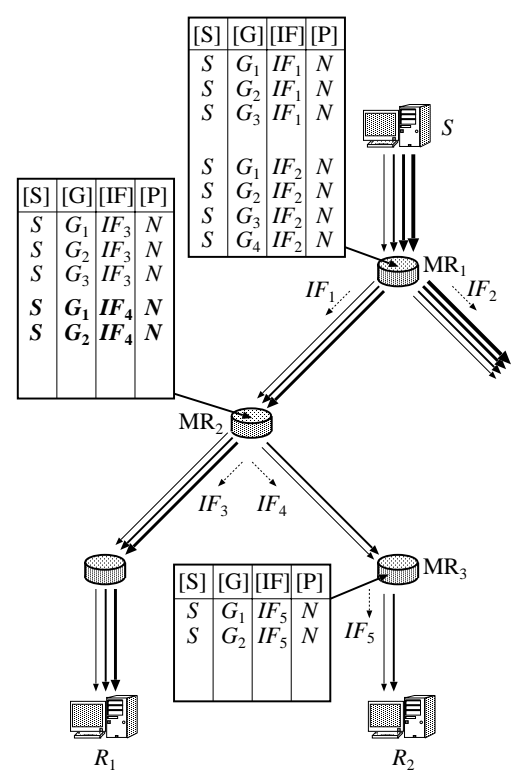

(e) $R_{2}$ receives stream with normal priority.

Fig. 3. Approach of the proposed scheme.

below a predefined threshold, MN transmits low priority join messages $\left\{\left(S, G_{i}\right), i=1, \ldots, n\right\}$ to the local multicast router through $\mathrm{IF}_{2}$, as shown in Fig. 4. MN then receives all layers $L_{i}$ with lower priority $\left(P_{i}-1\right)$ through $\mathrm{IF}_{2}$. After receiving the first packet of $L_{i}$, MN calculates $\rho_{i}$, the packet loss rate of $L_{i}$, for a time $T_{D}$. If $\rho_{k}$ exceeds a predefined threshold $\theta, \mathrm{MN}$ leaves multicast groups corresponding to layer $L_{k}$ and higher layers. Besides, it transmits normal join messages $\left\{\left(S, G_{i}\right)\right.$, $i=1, \ldots, k-1\}$ to the local multicast router, in order to join the lower layer with normal priority. After receiving the stream with normal priority, $\mathrm{MN}$ leaves the multicast groups through $\mathrm{IF}_{1}$ and shifts to $\mathrm{IF}_{2}$. Henceforth, it conducts join-experiment with lower priority through $\mathrm{IF}_{2}$ every $T_{J}$ period of time.

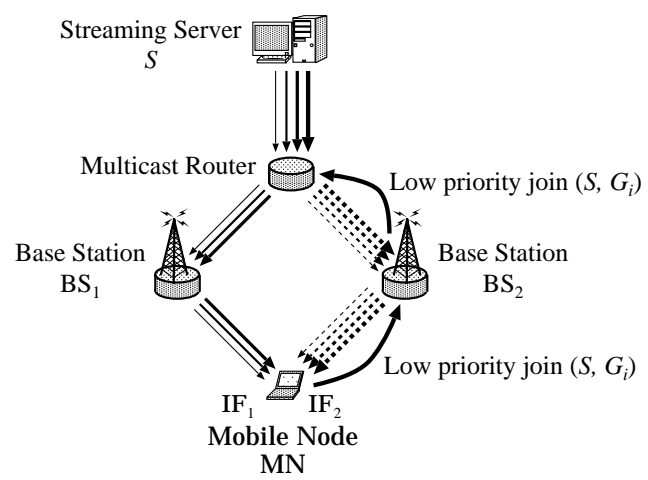

Fig. 4. Application of the proposed scheme to mobile networks. 


\section{Performance Evaluation}

In order to verify the effectiveness of our scheme, we implement the algorithm in the Network Simulator (ns-2.28) [16]. We carry out several simulations and compare it against RLM. This section gives a detailed description of the simulation environment and discusses the simulation results.

Similarly to [2], layered sources are modeled as a set of Constant Bit Rate (CBR) streams with fixed packet sizes (500 Bytes). Packets are generated at times defined as follows:

$$
T_{k}= \begin{cases}0 & \text { for } k=0 \\ T_{k-1}+\Delta+N_{k} & \text { for } k>0\end{cases}
$$

where $\Delta$ is a fixed interval deliberately chosen to meet the target bit rate. $N_{k}$ is uniformly distributed on $[-\Delta / 2, \Delta / 2]$. The traffic sources have twelve layers streamed at bit rates $B_{i}[\mathrm{kbps}]$ :

$$
B_{i}= \begin{cases}32 & \text { for } i=1 \\ 32 \cdot 2^{i-2} & \text { for } i \in\{2, \ldots, 12\}\end{cases}
$$

Cumulative bit rates range thus from $32 \mathrm{kbps}$ till $64 \mathrm{Mbps}$. They are exponentially spaced to simulate highly heterogeneous environments.

In our scheme, all routers need to support some priority disciplines as previously mentioned. In the conducted simulations, we use Weighted Random Early Detection (WRED) [17] for queuing. In WRED, while the average queue size is between a minimum threshold $T H_{\text {min }}$ and a maximum threshold $T H_{\max }$, the arriving packets are either dropped or queued, depending on the packet drop probability as shown in Fig. 5. If the average queue size is larger than $T H_{\text {max }}$, arriving packets are automatically dropped. Mark Probability Denominator $(M P D)$ indicates the fraction of packets discarded when the average queue depth is $T H_{\text {max }}$. WRED selectively discards lower priority traffic when a router begins to get congested. It provides also differentiated performance characteristics for different classes of service. We set the parameters of WRED as listed in Table II. Here, it should be emphasized that all routers in the network do not have to set the same parameter. On the other hand, RLM uses drop tail queue [2]. The maximum size of queues is set to 20 packets.

In the proposed scheme, we set both the initial value of $T_{J}$ $\left(T_{J}^{i n i t}\right)$ and $T_{D}$ to one second. The multiplicative coefficient $\alpha$,

TABLE II

WRED SETTING (UNIT: PACKETS)

\begin{tabular}{c|c|ccc}
\hline \hline \multirow{2}{*}{ Priority } & \multirow{2}{*}{ DSCP } & \multicolumn{3}{|c}{ WRED parameters [packets] } \\
\cline { 3 - 5 } & & $T H_{\min }$ & $T H_{\max }$ & $M P D$ \\
\hline 12 & AF41 & 11 & 15 & 20 \\
11 & AF42 & 10 & 14 & 16 \\
10 & AF43 & 9 & 13 & 13 \\
9 & AF31 & 8 & 12 & 10 \\
8 & AF32 & 7 & 11 & 9 \\
7 & AF33 & 6 & 10 & 8 \\
6 & AF21 & 5 & 9 & 7 \\
5 & AF22 & 4 & 8 & 6 \\
4 & AF23 & 3 & 7 & 5 \\
3 & AF11 & 2 & 6 & 4 \\
2 & AF12 & 1 & 5 & 3 \\
1 & AF13 & 0 & 4 & 2 \\
\hline \hline
\end{tabular}

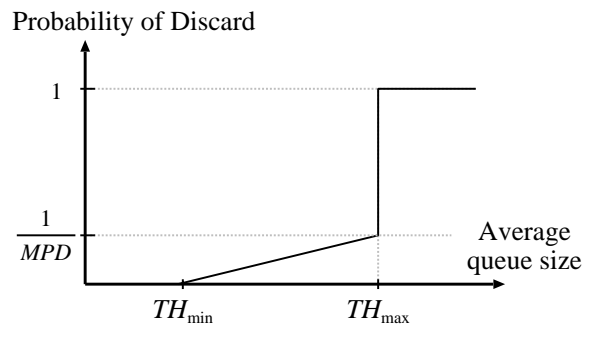

Fig. 5. Packet drop probability of WRED.

used in the computation of join timer $\left(T_{J}\right)$ (equation (3)), is set to two. RLM parameters are set to the same values as in [2]. The used multicast routing protocol is Distance Vector Multicast Routing Protocol (DVMRP). Both wired and wireless links are error-free throughout this paper.

Since the users cannot decode the higher layer without receiving all the lower layers in layered multicast, we use goodput to evaluate the performance of both schemes. Here, goodput is defined as throughput of the layers below the highest layer that users can sequentially achieve with less than $\theta=10$ [\%] of packet loss rate. This metric indicates the number of bytes received and actually decoded by users.

\section{A. Wired network}

To better understand the behavior of the proposed scheme, we first conduct simulations using a simple network topology made of only wired links as shown in Fig. 6. We consider a scenario where receivers $R_{1}$ and $R_{2}$ join a session provided by server $S_{1}$ at time $t=50$ [sec] and $t=200$ [sec], respectively. Fig. 7 plots the goodput transition of both users. In this scenario, $R_{1}$ and $R_{2}$ can receive data at a maximum bandwidth of $100 \mathrm{Mbps}$ and $10 \mathrm{Mbps}$, respectively. Fig. 7 indicates that both receivers succeeded in receiving data at appropriate rates, and that is the case for both schemes. However, it is observed that compared to the proposed scheme, RLM requires a significant time (over $100 \mathrm{sec}$ ) till it can make full utilization of the available network resources. The reason behind this performance consists in the fact that unlike RLM, our scheme allows users to estimate the available bandwidth by joining all layers with lower priority. In RLM, users need to conduct several join-experiments till they find out the available bandwidth. During this query for the available bandwidth,

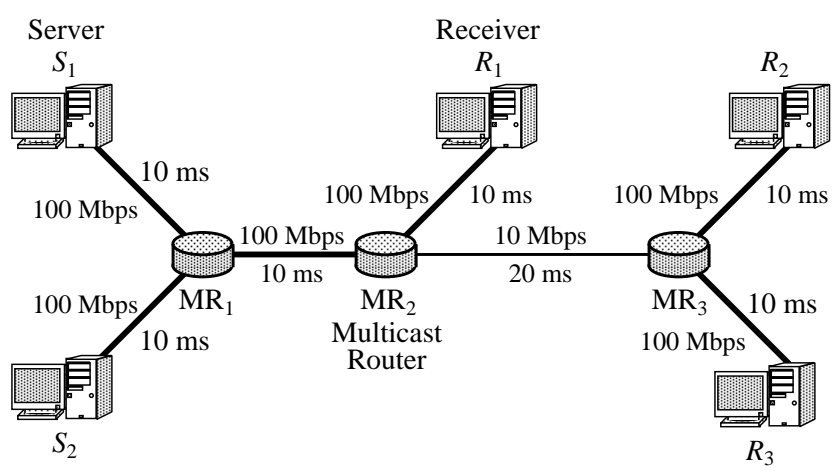

Fig. 6. A wired network topology. 


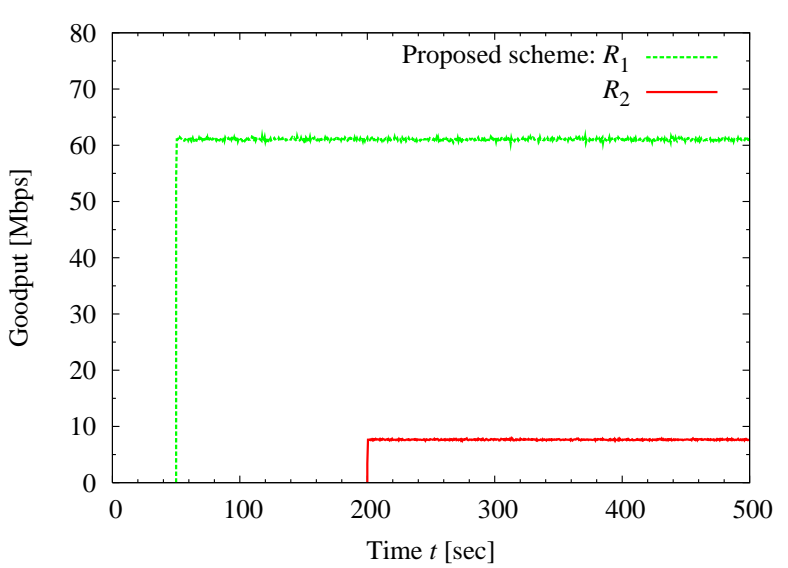

(a) Proposed scheme

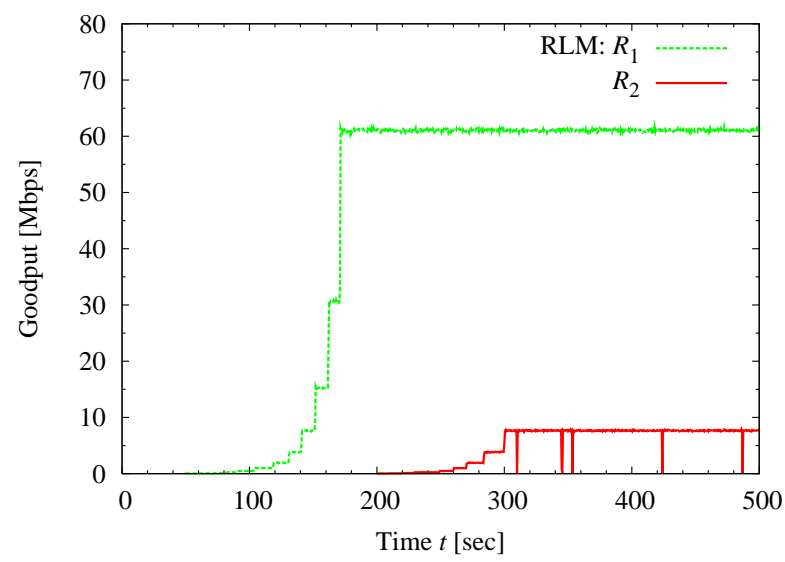

(b) RLM

Fig. 7. $R_{1}$ and $R_{2}$ join the same session at $t=50$ [sec] and $t=200$ [sec], respectively.

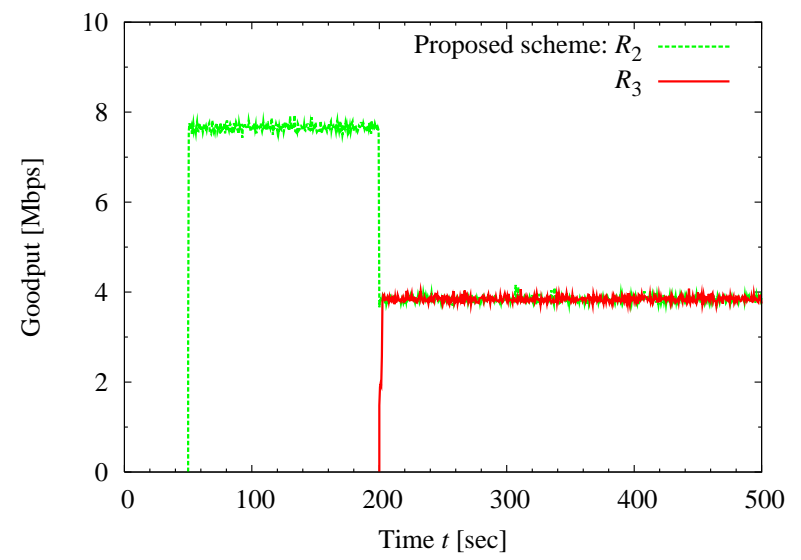

(a) Proposed scheme

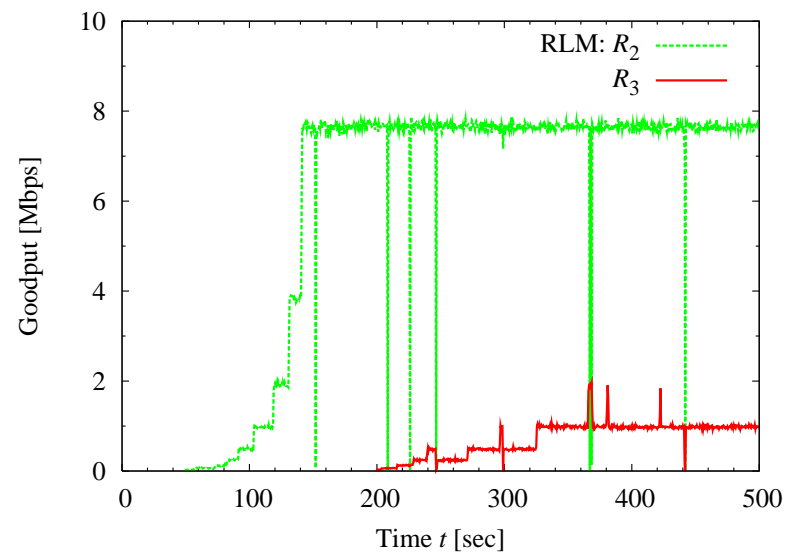

(b) RLM

Fig. 8. $R_{2}$ and $R_{3}$ join two different sessions at $t=50$ [sec] and $t=200$ [sec], respectively.

significant packet drops occur. On the other hand, in the proposed scheme, users conduct join-experiments at only one time with lower priority. Accordingly, users can receive data at appropriate bit rates from the beginning of the session.

To investigate the performance of our scheme when two different sessions share the same bottleneck link, we consider a scenario where $R_{2}$ joins a session from server $S_{1}$ at time $t=50$ [sec] and $R_{3}$ joins another session from another server $S_{2}$ at $t=200$ [sec]. The results are plotted in Fig. 8. The figure demonstrates that RLM results in strong unfairness between the two users. Indeed, it shows that $R_{2}$ conquers most of the available bandwidth when RLM is in use. On the other hand, in case of the proposed scheme, the figure indicates that both users share the network resources in a fair manner. This performance is attributable to the priority dropping mechanism of the proposed scheme. Observe also that both users experience some oscillations in their goodput transition in case of RLM whereas their goodputs remain stable in case of the proposed scheme. The reason behind this performance underlies beneath the fact that users perform joinexperiments with lower priority in the proposed scheme.

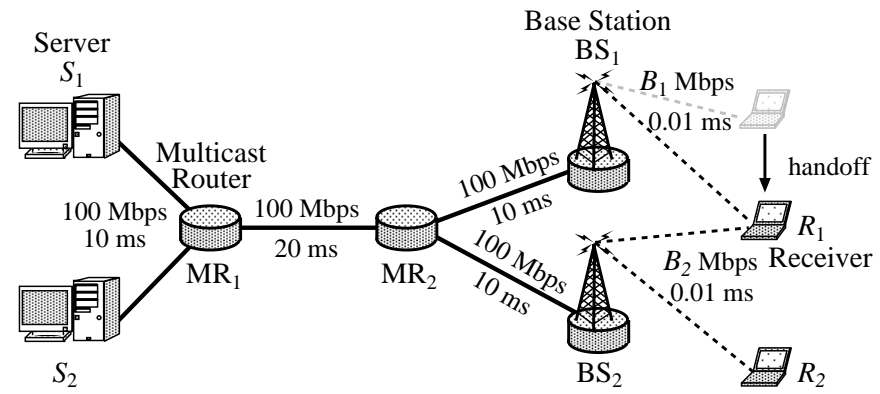

Fig. 9. Hybrid wired/wireless network topology.

\section{B. Hybrid wired/wireless network}

In the remainder of this section, we investigate the performance of our scheme in mobile environments. At the beginning of the simulation, a mobile node $R_{1}$ resides in the cell of $\mathrm{BS}_{1}$. It then moves into the cell overlapping area of $\mathrm{BS}_{1}$ and $\mathrm{BS}_{2}$, and performs handoff as shown in Fig. 9. In this simulation, two scenarios are envisioned: the mobile node moves from a lower bandwidth cell to a higher bandwidth cell or vice versa. Figs. 10 and 11 graph the goodput 


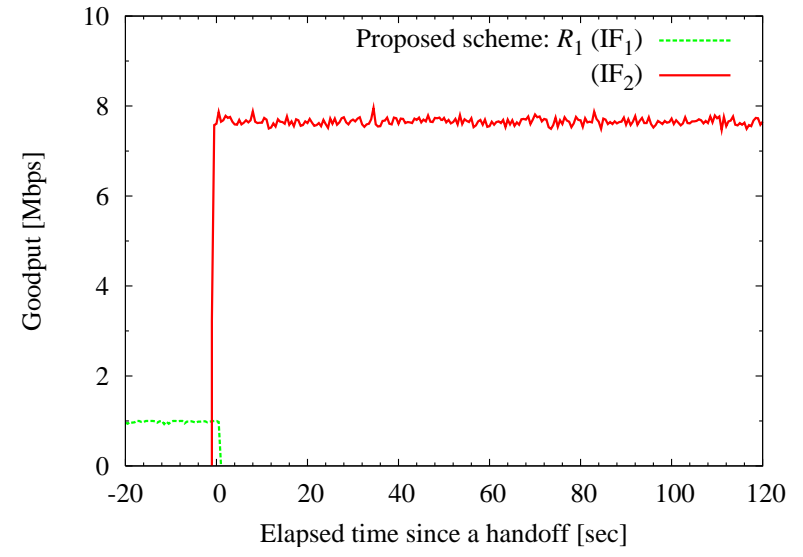

(a) Proposed scheme

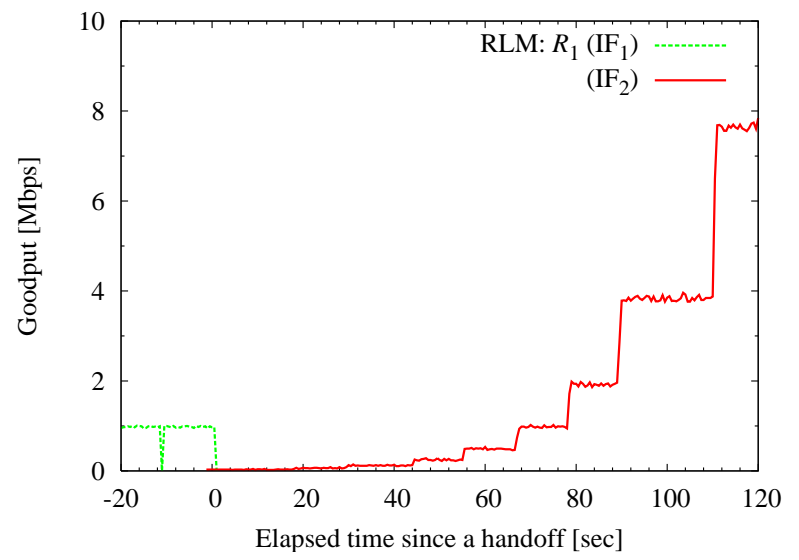

(b) RLM

Fig. 10. $R_{1}$ performs handoff from $1 \mathrm{Mbps}$ to $11 \mathrm{Mbps}$ when only a single session exists.

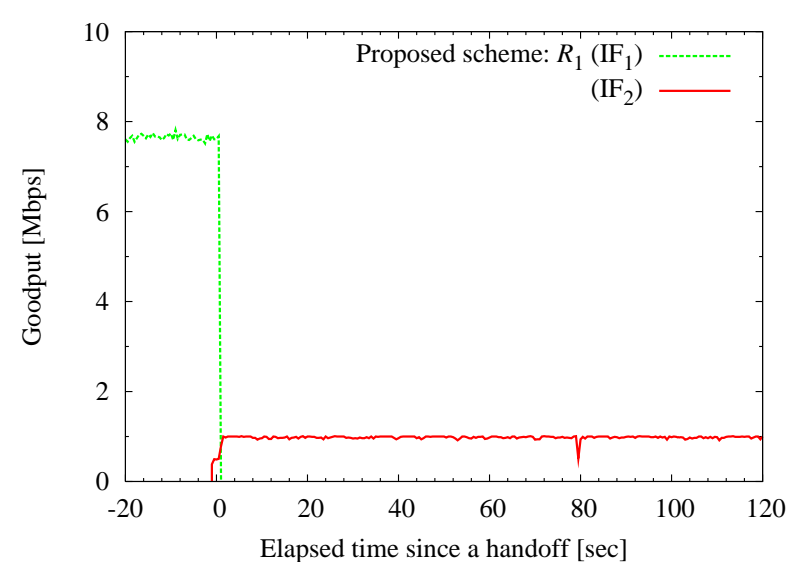

(a) Proposed scheme

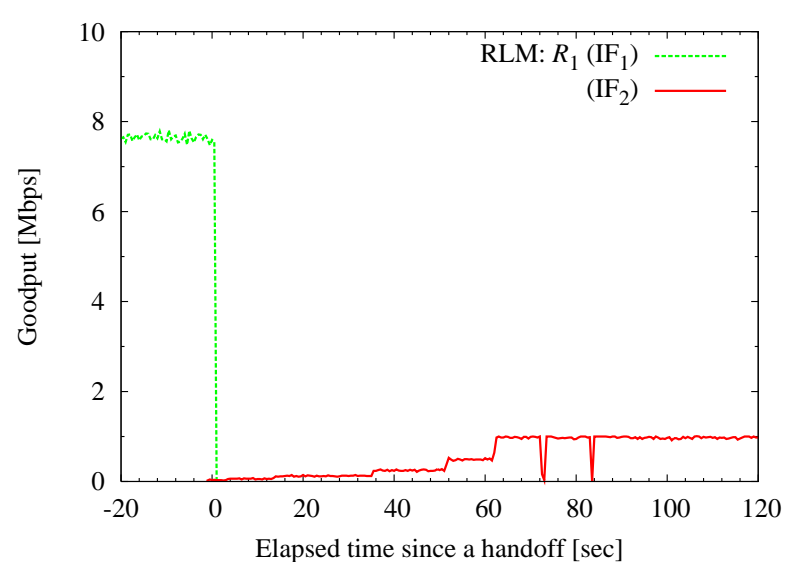

(b) RLM

Fig. 11. $R_{1}$ performs handoff from $11 \mathrm{Mbps}$ to $1 \mathrm{Mbps}$ when only a single session exists.

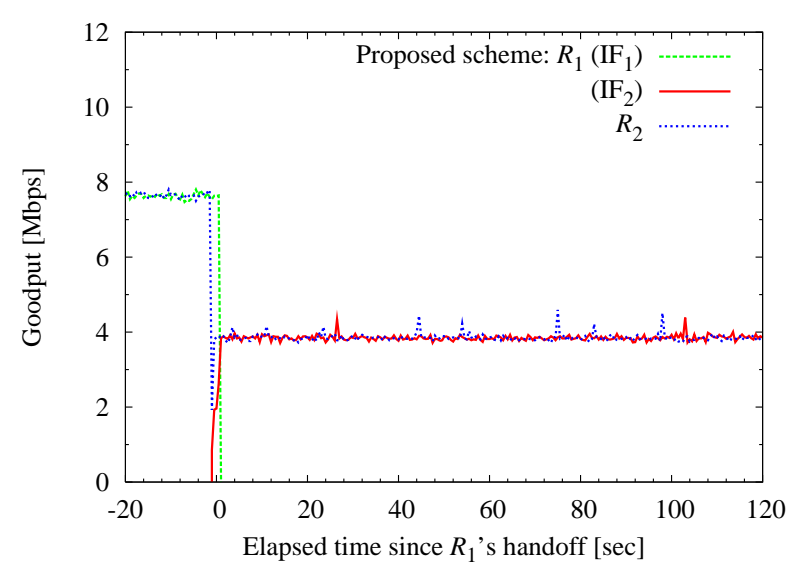

(a) Proposed scheme

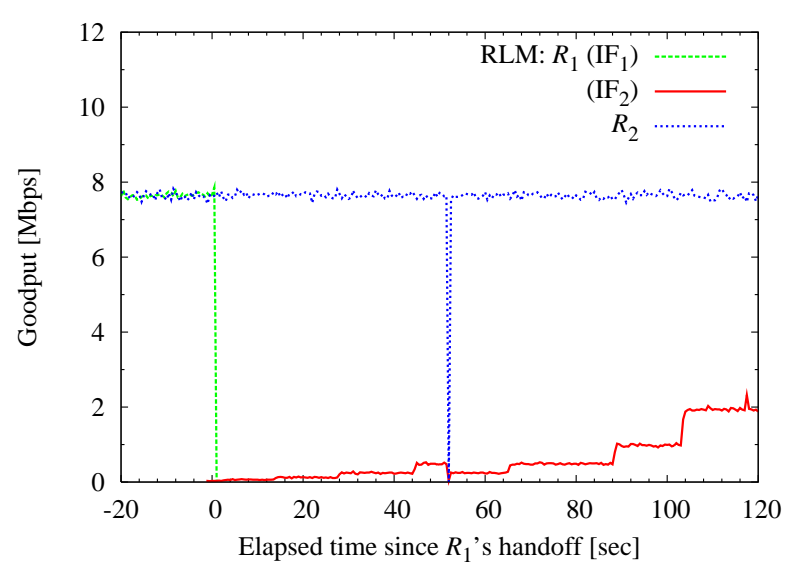

(b) RLM

Fig. 12. $R_{1}$ performs handoff from $\mathrm{BS}_{1}$ to $\mathrm{BS}_{2}$ and shares $11 \mathrm{Mbps}$ with $R_{2}$ after handoff. 
transition when receiver $R_{1}$ performs handoff from $1 \mathrm{Mbps}$ network to $11 \mathrm{Mbps}$ network (i.e. $B_{1}=1[\mathrm{Mbps}], B_{2}=$ 11 [Mbps]), and from $11 \mathrm{Mbps}$ to $1 \mathrm{Mbps}$, respectively. These graphs demonstrate that the proposed scheme enables $R_{1}$ to receive the stream at rates suitable to the available bandwidth immediately after handoff. On the other hand, RLM needs longer convergence time as it adds the upper layers one-byone.

Next, we evaluate the performance of the proposed scheme when receiver $R_{1}$ performs handoff and shares the available bandwidth of the new network with another user joining a different session. At the beginning of the simulation, receivers $R_{1}$ and $R_{2}$ reside in cells of $\mathrm{BS}_{1}$ and $\mathrm{BS}_{2}$, respectively. $R_{1}$ then moves into the cell overlapping area, performs handoff, and starts sharing the cell resources with $R_{2}$. Bandwidth of the two cells is set to $11 \mathrm{Mbps}$. Fig. 12 shows the goodput transition of both receivers. It is clear that the proposed scheme succeeds in fairly dividing the bandwidth of the new network between the two receivers immediately after $R_{1}$ 's handoff. However, in case of RLM, $R_{2}$ remains conquering most of the network resources. This unfairness issue can be explained in the same way as when users share the bandwidth of the same bottleneck link.

\section{CONCLUSION}

In this paper, we proposed a layered multicast streaming scheme. The proposed scheme exploits the fact that layered multicast uses priority-based packet dropping policies. In our method, a user joins all layers with lower priority at the beginning of a session. He/she then calculates the packet loss rate on each layer. If the packet loss rate on a certain layer exceeds a predefined threshold, the user leaves that layer and higher layers. The user then receives packets of lower layers with normal priority. By so doing, users can converge fast to the optimal bit rate (most suitable rate to the network conditions) at the beginning of a session. Additionally, as they conduct join-experiments with lower priority at regular time intervals, users are always aware of the bandwidth availability of the underlying network without dropping packets of lower layer.

The performance of the proposed scheme was investigated through several simulations. Performance evaluation relied on computer simulation. The obtained results revealed that our scheme enables users to fairly share the bandwidth with other users. It achieves appropriate streaming rate from the beginning of the session. The efficiency of the proposed scheme in mobile networks is also confirmed. Indeed, our scheme guarantees smooth handoff and enables mobile nodes to receive streams at rates appropriate to network conditions immediately after handoff occurrence. The good fairness of the proposed scheme in mobile environments is also verified.

\section{REFERENCES}

[1] N. Shacham, "Multipoint Communication by Hierarchically Encoded Data," in Proc. of IEEE INFOCOM '92, Florence, Italy, May 1992, pp. $2107-2114$.

[2] S. McCanne, V. Jacobson, and M. Vetterli, "Receiver-driven Layered Multicast," in Proc. of ACM SIGCOMM '96, Stanford, CA, USA, Aug. 1996, pp. $117-130$.
[3] S. Bajaj, L. Breslau, and S. Shenker, "Uniform versus Priority Dropping for Layered Video," in Proc. of ACM SIGCOMM '98, Vancouver, BC, Canada, Sept. 1998, pp. 131-143.

[4] C.-K. Tham, Y.-S. Gan, and Y. Jiang, "Congestion Adaptation and Layer Prioritization in a Multicast Scalable Video Delivery System," in Proc. of IEEE/EURASIP Packet Video 2003, Nantes, France, Apr. 2003.

[5] S. Y. Cheung, M. H. Ammar, and X. Li, "On the use of destination set grouping to improve fairness in multicast video distribution," in Proc. of IEEE INFOCOM '96, San Francisco, CA, USA, Mar. 1996, pp. 553560.

[6] X. Li, M. Ammar, and S. Paul, "Video Multicast over the Internet," IEEE Network, vol. 13, no. 2, pp. 46-60, Mar./Apr. 1999.

[7] E. Amir, S. McCanne, and H. Zhang, "An Application Level Video Gateway," in Proc. of ACM Multimedia '95, San Francisco, CA, USA, Nov. 1995 , pp. 255-265.

[8] A. Legout and E. W. Biersack, "PLM: Fast Convergence for Cumulative Layered Multicast Transmission Schemes," in Proc. of ACM SIGMETRICS '2000, Santa Clara, CA, USA, June 2000, pp. 13-22.

[9] Y. Pan, M. Lee, J. B. Kim, and T. Suda, "An End-to-End Multipath Smooth Handoff Scheme for Stream Media," IEEE J. Select. Areas Commun., vol. 22, no. 4, pp. 653-663, May 2004.

[10] G. Cunningham, S. Murphy, P. Perry, and L. Murphy, "Seamless Handover of Streamed Video over UDP between Wireless LANs," in Proc. of IEEE CCNC 2005, Las Vegas, NV, USA, Jan. 2005, pp. 284289.

[11] J. Heinanen, F. Baker, W. Weiss, and J. Wroclawski, "Assured Forwarding PHB Group," RFC 2597, June 1999.

[12] R. Chandra, Paramvir Bahl, and Pradeep Bahl, "MultiNet: Connecting to Multiple IEEE 802.11 Networks Using a Single Wireless Card," in Proc. of IEEE INFOCOM 2004, vol. 2, Hong Kong, China, Mar. 2004, pp. 882-893.

[13] H. Schulzrinne, S. Casner, R. Frederick, and V. Jacobson, "RTP: A Transport Protocol for Real-Time Applications," RFC 3550, July 2003

[14] T. Taleb, K. Kashibuchi, N. Kato, and Y. Nemoto, "A Dummy Segment Based Bandwidth Probing Technique to Enhance the Performance of TCP over Heterogeneous Networks," in Proc. of IEEE WCNC 2005, vol. 4, New Orleans, RA, USA, Mar. 2005, pp. 2400-2405.

[15] K. Kashibuchi, T. Taleb, A. Jamalipour, Y. Nemoto, and N. Kato, "A New Smooth Handoff Scheme for Mobile Multimedia Streaming using RTP Dummy Packets and RTCP Explicit Handoff Notification," in Proc. of IEEE WCNC 2006, Las Vegas, NV, USA, Apr. 2006.

[16] The Network Simulator - ns-2. [Online]. Available: http://www.isi.edu/ nsnam/ns/

[17] Cisco IOS Quality of Service Solutions Configuration Guide, Release 12.2. [Online]. Available: http://www.cisco.com/univercd/cc/td/doc/ product/software/ios $122 / 122 \mathrm{cgcr} / \mathrm{fqos}$ c c/qcfbook.pdf

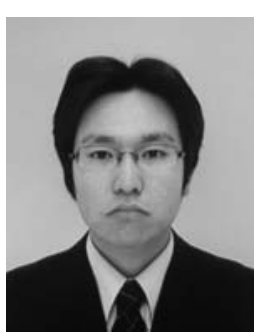

Kenichi Kashibuchi received his B.E degree in information engineering from Tohoku University, Sendai, Japan, in 2005. He is currently working towards his M.S degree at the Graduate School of Information Sciences, Tohoku University. His current research interests are in hybrid wired/wireless multimedia systems and mobile networking, especially in the performance of transport protocols during handoff. He is an IEEE student member. 


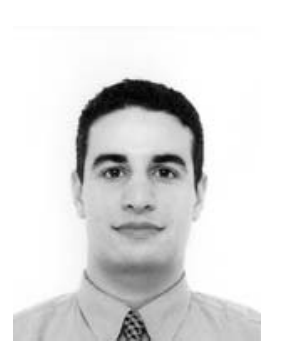

Tarik Taleb is currently working as assistant professor with the Graduate School of Information Sciences (GSIS), Tohoku University, Japan. From Oct. 2005 till Mar. 2006, he was working as research fellow with the Intelligent Cosmos Research Institute, Sendai, Japan. He received his B.E degree in Information Sciences with distinction, M.E and Ph.D degrees in Computer Sciences from GSIS, Tohoku Univ., in 2001, 2003, and 2005, respectively. His research interests lie in the field of wireless networking, inter-vehicular communications, satellite and space communications, congestion control protocols, and network security. His recent research has focused also on on-demand media transmission in multicast environments and mobility management. Dr. Taleb has been on the technical program committee of different IEEE conferences, including ICC, GLOBECOM, and WCNC, and chaired some of their sessions. He has acted also as reviewer for many IEEE conferences, IEICE Transactions on Communications journal, and IEEE/ACM Transactions on Networking journal. He is a recipient of the Niwa Yasujirou Memorial award (Feb. 2005) and the Young Researcher's Encouragement award from the Japan chapter of the IEEE Vehicular Technology Society (VTS) (Oct. 2003). Dr. Taleb is an IEEE member.

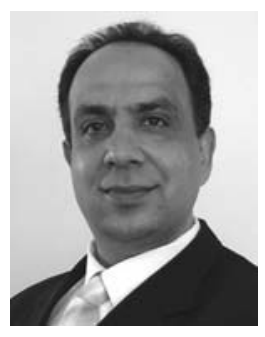

Abbas Jamalipour (S'86, M'91, SM'00) has been on the faculty of Sydney University, Australia since 1998. He holds a Ph.D from Nagoya University, Japan. He is the author of the first book on wireless IP and two other books, and has co-authored five books and over 150 journal and conference papers, all in the field of wireless telecommunications. Professor Jamalipour is an IEEE Distinguished Lecturer, the Editor-in-Chief IEEE Wireless Communications, and a Fellow Member of IEAust. He has been very active within the ComSoc, currently serving as the Chair of Satellite and Space Communications TC; Vice Chair of Communications Switching and Routing TC; and Chair of Coordinating Committee Chapter, Asia-Pacific Board. He is a Technical Editor of the Communications Magazine, the Wiley International Journal of Communication Systems, and several other scholarly journals. He is a voting member of GITC and has been a Vice Chair of WCNC2003 to 2006, Chair of GLOBECOM2005 (Wireless Communications), and a symposium Co-Chair of ICC2005 to 2007, and GLOBECOM2006, and many other conferences in the past.

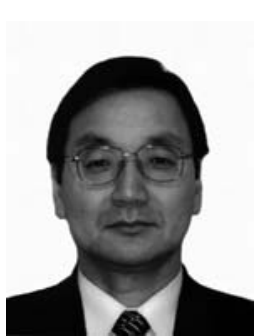

Yoshiaki Nemoto received his B.E., M.E., and $\mathrm{Ph} . \mathrm{D}$. degrees from Tohoku University in 1968, 1970, and 1973, respectively. He is a full professor with the Graduate School of Information Sciences, and served as director of the Information Synergy Center, Tohoku University. He has been engaged in research work on microwave networks, communication systems, computer network systems, image processing, and handwritten character recognition. $\mathrm{He}$ is a recipient of the 2005 Distinguished Contributions to Satellite Communications award from IEEE ComSoc society and a co-recipient of the 1982 Microwave Prize from the IEEE MTT society. He is a senior member of IEEE, a member of IEICE, and a fellow member of the Information Processing Society of Japan.

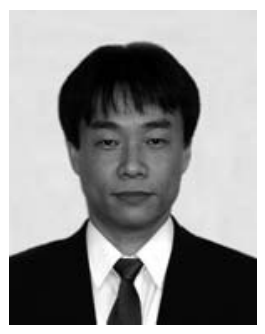

Nei Kato received his M.S. and Ph.D. degrees from the Graduate School of Information Sciences, Tohoku University, in 1988 and 1991, respectively. He has been working for Tohoku University since then and is currently a full professor at the Graduate School of Information Sciences. He has been engaged in research on computer networking, wireless mobile communications, image processing, and neural networks. He is a member of the Institute of Electronics, Information and Communication Engineers (IEICE) of Japan and a senior member of IEEE. He has served on a large number of technical program and organizing committees of international conferences. From 2006, he is serving as a technical editor of IEEE Wireless Communications. 